\title{
Simultaneous and Rapid Determination of Six Tyrosine Kinase Inhibitors in Patients with Non-Small Cell Lung Cancer Using HPLC-MS/MS
}

\author{
Yanping Liu, ${ }^{1,2}$ Hua Liu, ${ }^{3}$ Zhewei Xia, ${ }^{4}$ Zhipeng Wang ${ }^{(D},{ }^{1}$ Yunlei Yun, ${ }^{1}$ Guanying Zhang, ${ }^{2}$ \\ Lifeng Huang $\left(\mathbb{D},{ }^{2}\right.$ Shouhong Gao $\mathbb{i}{ }^{1},{ }^{1}$ and Wansheng Chen $(\mathbb{1})^{1}$ \\ ${ }^{1}$ Department of Pharmacy, Changzheng Hospital, Second Military Medical University, Shanghai 200003, China \\ ${ }^{2}$ Department of Pharmacy, The Affliated Suzhou Science and Technology Town Hospital of Nanjing Medical University, \\ Suzhou 215153, China \\ ${ }^{3}$ Department of Pharmacy, Taixing People's Hospital, Taizhou, Jiangsu 225400, China \\ ${ }^{4}$ Pharmaceutical Analysis and Testing Center, School of Pharmacy, The Second Military Medical University, \\ Shanghai 200003, China \\ Correspondence should be addressed to Lifeng Huang; huanglifeng@yeah.net, Shouhong Gao; gaoshouhong@smmu.edu.cn, and \\ Wansheng Chen; chenwansheng@smmu.edu.cn
}

Received 23 February 2021; Accepted 13 August 2021; Published 17 September 2021

Academic Editor: Mohamed Abdel-Rehim

Copyright (c) 2021 Yanping Liu et al. This is an open access article distributed under the Creative Commons Attribution License, which permits unrestricted use, distribution, and reproduction in any medium, provided the original work is properly cited.

Objective. To develop a new method for quantitatively analyzing six tyrosine kinase inhibitors (gefitinib, erlotinib, icotinib, afatinib, osimertinib, and crizotinib) used in the treatment of non-small cell lung cancer (NSCLC) by liquid chromatographytandem mass spectrometry (LC-MS/MS). Methods. The analytes were detected in the selected reaction monitoring mode on a triple quadrupole mass spectrometer with the positive ionization mode. Carbamazepine was utilized as the internal standard. The pretreatment of the plasma sample was completed based on protein precipitation with acetonitrile, and the analytes were separated on an Agilent Zorbax SB-C18 reversed-phase column $(2.1 \mathrm{~mm} \times 100 \mathrm{~mm}, 3.5 \mu \mathrm{m}$, Agilent, USA) using gradient elution. The mobile phase consisted of $0.1 \%$ formic acid in water (phase A) and $0.1 \%$ formic acid in acetonitrile (phase B). The flow rate was $0.3 \mathrm{~mL} / \mathrm{min}$, and the injection volume was $5 \mu \mathrm{L}$. The column temperature was set and maintained at $35^{\circ} \mathrm{C}$. Results. The calibration curves were linear over the range from 5.0 to $1000.0 \mathrm{ng} / \mathrm{mL}$ for gefitinib, crizotinib, and osimertinib; from 50.0 to $4000.0 \mathrm{ng} / \mathrm{mL}$ for icotinib and erlotinib; and from 5.0 to $400.0 \mathrm{ng} / \mathrm{mL}$ for afatinib. Linear correlation coefficients were $>0.990$ for all regression curves. The intra- and interday accuracy and precision of the method were within $\pm 15.0 \%$ and not more than $15.0 \%$, respectively. The mean recovery of all the analytes ranged from $70.18 \%$ to $110.76 \%$, the matrix effect was from $88.85 \%$ to $127.58 \%$, and stability was within $\pm 15.0 \%$. Conclusion. This newly developed method was sensitive, simple, and robust and could be used in therapeutic drug monitoring of six tyrosine kinase inhibitors in NSCLC patients.

\section{Introduction}

Lung cancer is one of the most common malignant tumors in the world, among which the number of patients with nonsmall cell lung cancer (NSCLC) is about $85-90 \%$, and $14 \%$ of cancer deaths in the world are caused by NSCLC [1]. In the last 50 years, treatments for NSCLC mainly included chemical drugs. Chemotherapy regimens of cisplatin, vincristine combined with cisplatin, and paclitaxel have prolonged the survival time and improved the quality of life of lung cancer patients, but the 5-year overall survival rate is about 5\% [2]. In recent years, tumor treatment has developed rapidly, and oral small-molecule targeting drugs have emerged successively. These oral small-molecule targeting drugs bind to certain receptors on the tumor cells, thereby inhibiting downstream tyrosine kinase signaling and tumor proliferation. The primary small-molecule target drugs mainly target epidermal growth factor receptor (EGFR) and 
anaplastic lymphoma kinase (ALK). EGFR and ALK mutations occur mainly in nonsmoking NSCLC patients, and nonsmoking patients also have higher survival rates than smokers [3]. In newly treated patients with EGFR-mutated NSCLC, compared with platinum chemotherapy, firstgeneration EGFR inhibitors (gefitinib and erlotinib) or second-generation EGFR inhibitors (afatinib and icotinib) had significant higher survival and better quality of life [4]. However, EGFR (T790M) secondary mutations occur in approximately $50 \%$ of patients with progressive lung cancer after 9-13 months of treatment. Osimertinib, the thirdgeneration EGFR inhibitor, showed a greater survival advantage in patients with secondary mutations at EGFR (T790M) than platinum-based chemotherapy [5]. Crizotinib significantly improved the quality of life in patients of nonprogressive lung cancer or NSCLC of ALK rearrangement [6].

Molecular targeted drugs are all given orally on fixed dose, which have good efficacy, but there are also many adverse reactions. Adverse reactions are one of the factors leading to interruption of treatment. Many pharmacokinetic studies have reported the relationship between clinical efficacy or adverse reactions and plasma exposure [7-10]. However, there were significant individual differences in plasma exposure levels. The therapeutic window of these drugs was narrow, and there were significant differences in pharmacokinetics among individual patients [11]; therefore, these drugs are candidates for therapeutic drug monitoring (TDM). TDM is a dose optimization strategy to achieve faster and more effective clinical efficacy. The use of the rapid and sensitive LC-MS/MS detection method to determine the concentration of drugs in human blood or other body fluids can effectively adjust the dosage of drugs, improve the therapeutic effect of drugs, reduce the adverse reactions, and ensure the rational use of drugs. In order to solve this problem, several LC-MS/MS methods have been developed [12-15], but these methods have high requirements for instruments, complicated operation, and long analysis time, which hinder the clinical application. Therefore, the purpose of this study was to establish a simple, rapid, and sensitive method for the simultaneous determination of six TKIs in human plasma and verify its clinical application.

\section{Materials and Methods}

2.1. Chemicals and Reagents. The analytes including gefitinib (lot: F1102AS), icotinib (lot: J0615A), afatinib (lot: M0320A), erlotinib (lot: J0615A), crizotinib (lot: A0320A), and carbamazepine (internal standard, IS) (lot: M1001AS) were supplied by Meilun Biotech Co., Ltd. (Dalian City, China). Osimertinib (lot: 1-NJL-79-1) was purchased from Toronto Research Chemicals (Toronto, Canada). Mass spectrometric reagents methanol and acetonitrile were obtained from Merck (Merck Company, Darmstadt, Germany).

Formic acid was purchased from Tedia Company Inc. (Tedia, Fairfield, OH, USA). Isopropanol reagent was purchased from Shanghai Titan Technology Co., Ltd. (Titan, Shanghai, China). Distilled water was purchased from
Watsons Distilled Water Co., Ltd. (Watsons, Guangzhou, China). Human blank plasma was donated by healthy volunteers in our laboratory (Shanghai, China).

2.2. Mass Spectrometry. The experiment was performed on an Agilent 1200 series HPLC system, consisting of an online degasser, a binary pump, an autosampler, and a column oven and interfaced to an Agilent 6410A triple quadrupole mass spectrometer equipped, in which the ionization source is the electrospray ionization source (ESI source, Agilent Technologies, USA). The data were processed using Agilent MassHunter data processing software (version B.01.04; Agilent Technologies, USA).

2.3. Liquid Chromatographic Conditions. All analytes were performed on a Zorbax SB-C18 analytical column $(2.1 \mathrm{~mm} \times 100 \mathrm{~mm}, 3.5 \mu \mathrm{m}$, Agilent, USA). The mobile phases contained $0.1 \%$ formic acid in water (phase A) and $0.1 \%$ formic acid in acetonitrile (phase B), and the flow rate was set at $0.3 \mathrm{~mL} / \mathrm{min}$. The gradient program started at $30 \%$ B, increased gradually to $90 \%$ B in $3 \mathrm{~min}$, and then was held at $90 \% \mathrm{~B}$ until $7 \mathrm{~min}$. The pastime was $5 \mathrm{~min}$. The column temperature was set at $35^{\circ} \mathrm{C}$, and the autosampler was maintained at room temperature. The volume injected into the chromatographic system was $5 \mu \mathrm{L}$.

2.4. Mass Spectrometry Conditions. All analytes were collected under the multiple reaction monitoring (MRM) and positive ionization mode (Figure 1). The mass spectrum parameters were as follows: HPLC flow rate of $250 \mathrm{~L} / \mathrm{min}$, sheath gas flow rate of $12 \mathrm{~L} / \mathrm{min}$, and temperature of $250^{\circ} \mathrm{C}$. Nozzle voltage is $500 \mathrm{~V}$. The atomizer pressure is $45 \mathrm{psi}$. Capillary voltage is $4000 \mathrm{~V}$. The drying gas and atomizing gas are nitrogen, the flow rate of drying gas is $5 \mathrm{~L} / \mathrm{min}$, and the temperature is $350^{\circ} \mathrm{C}$. Table 1 shows the optimized MRM parameters for six analytes and IS.

2.5. Preparation of Standard and Quality Control Samples. The stock solutions of all analytes were prepared, respectively, in $70 \%$ methanol (methanol-water, $70: 30, \mathrm{~V} / \mathrm{V}$ ), and $2.05,2.02,1.99,2.02,2.08$, and $2.00 \mathrm{mg}$ of gefitinib, icotinib, afatinib, erlotinib, crizotinib, and osimertinib were accurately weighed and dissolved to obtain $1.0 \mathrm{mg} /$ $\mathrm{mL}$ for all of them. The stock solutions were aliquoted and stored at $-80^{\circ} \mathrm{C}$. The stock solution of analytes was further diluted with $10 \%$ methanol (methanol-water, $10: 90, \mathrm{~V} / \mathrm{V}$ ) to obtain combined work solutions at the following concentrations: $100,200,500,1000,2000,5000$, and $10000 \mathrm{ng} / \mathrm{mL}$ for gefitinib, crizotinib, and osimertinib; 500, 1000, 2000, 5000, 10000,20000 , and $40000 \mathrm{ng} / \mathrm{mL}$ for icotinib and erlotinib; and $50,100,200,500,1000,2000$, and $4000 \mathrm{ng} / \mathrm{mL}$ for afatinib; they were diluted with blank human plasma 10 times, and their concentrations ranged from 10 to $1000 \mathrm{ng} / \mathrm{mL}$ for gefitinib, crizotinib, and osimertinib; from 50 to $4000 \mathrm{ng} / \mathrm{mL}$ for icotinib and erlotinib; and from 5 to $400 \mathrm{ng} / \mathrm{mL}$ for afatinib. Quality control (QC) samples were also prepared in the same way for each TK, and their concentrations were set at 


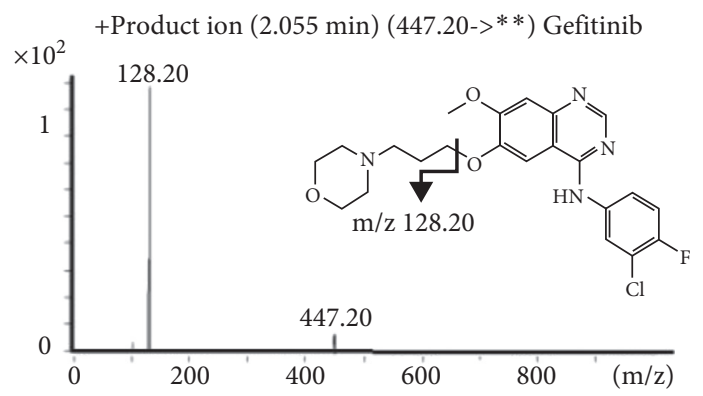

(a)

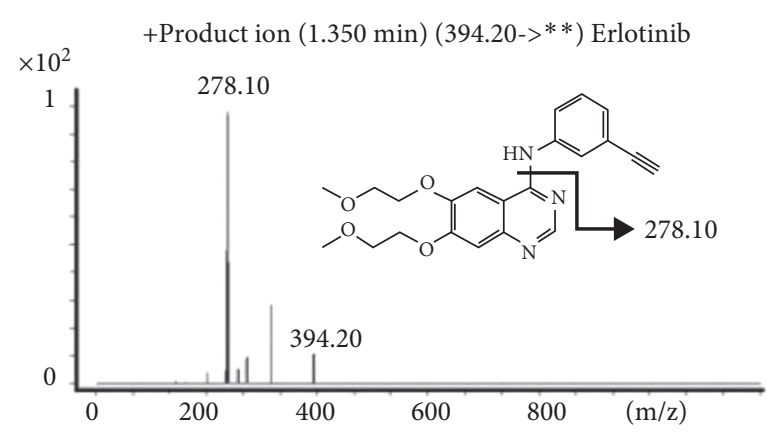

(c)

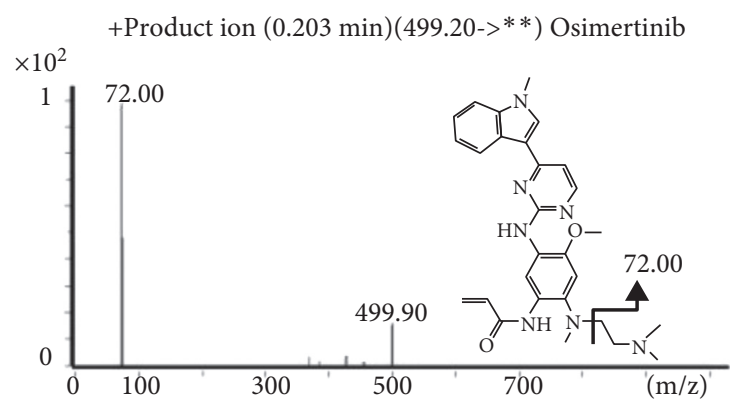

(e)

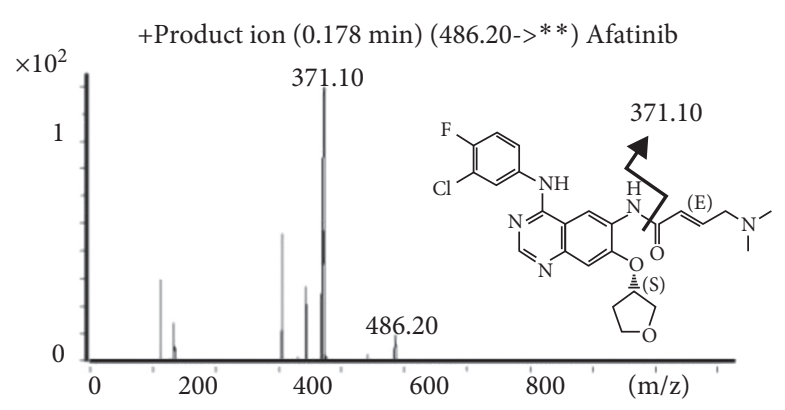

(b)

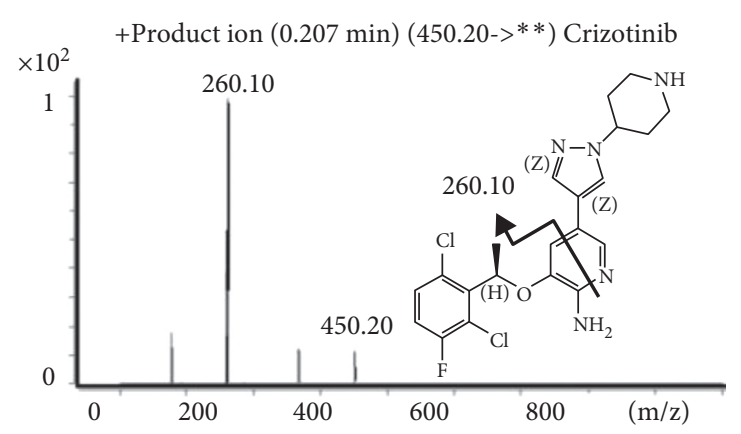

(d)

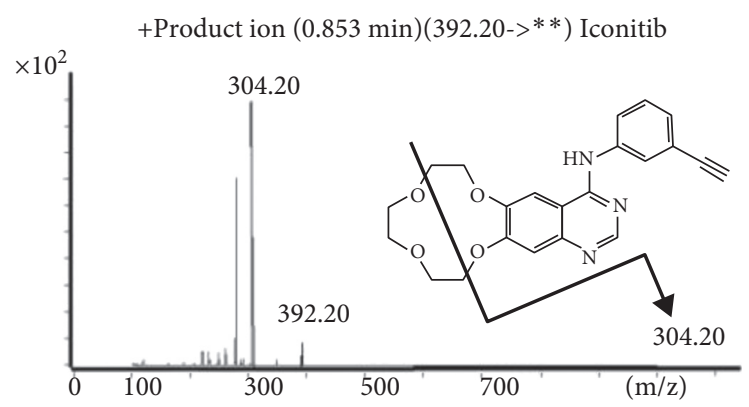

(f)

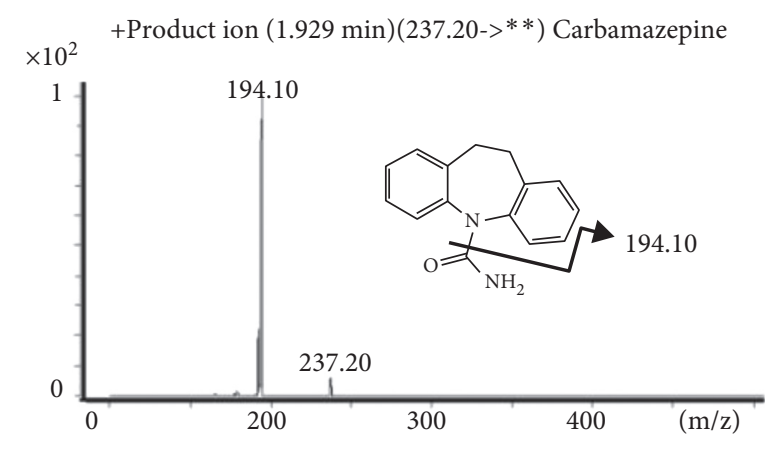

(g)

Figure 1: Product ion chromatograms and fragment structures of six TKIs. (a) Gefitinib. (b) Afatinib. (c) Erlotinib. (d) Crizotinib. (e) Osimertinib. (f) Icotinib. (g) Carbamazepine (IS).

20.0, 100.0, and $500.0 \mathrm{ng} / \mathrm{mL}$ for gefitinib, crizotinib, and osimertinib; at 100.0, 500.0, and $2000.0 \mathrm{ng} / \mathrm{mL}$ for icotinib and erlotinib; and at $10.0,50.0$, and $200.0 \mathrm{ng} / \mathrm{mL}$ for afatinib. The stock solutions were stored at $-80^{\circ} \mathrm{C}$. They were brought to room temperature $\left(25^{\circ} \mathrm{C}\right)$ for thaw before pretreatment. For IS stock solution, $1.99 \mathrm{mg}$ carbamazepine was dissolved in $70 \%$ methanol and stored at $-80^{\circ} \mathrm{C}$ after aliquot. The IS work solution was freshly prepared with acetonitrile at a concentration of $100 \mathrm{ng} / \mathrm{mL}$ for carbamazepine and stored at $-20^{\circ} \mathrm{C}$.

2.6. Sample Pretreatment. The samples were prepared as follows: for all analytes, sample pretreatment was performed by protein precipitation. The blood sample $(50 \mu \mathrm{L})$ was 
TABLE 1: Optimized mass spectrometry parameters of six analytes and IS.

\begin{tabular}{|c|c|c|c|c|c|}
\hline Analytes & Ionization mode $( \pm)$ & Precursor ions $(\mathrm{m} / \mathrm{z})$ & Fragmentor (V) & Collision energy (V) & Product ions $(\mathrm{m} / \mathrm{z})$ \\
\hline Gefitinib & + & 447.2 & 90 & 22 & 128.2 \\
\hline Icotinib & + & 392.1 & 155 & 32 & 304.2 \\
\hline Afatinib & + & 486.2 & 140 & 32 & 371.1 \\
\hline Erlotinib & + & 394.2 & 100 & 30 & 278.1 \\
\hline Crizotinib & + & 450.2 & 140 & 26 & 260.1 \\
\hline Osimertinib & + & 499.9 & 105 & 26 & 72.0 \\
\hline Carbamazepine (IS) & + & 237.1 & 115 & 19 & 194.1 \\
\hline
\end{tabular}

transferred to a $1.5 \mathrm{~mL}$ Eppendorf tube prior to spiking with $100 \mu \mathrm{L}$ of acetonitrile (containing $100 \mathrm{ng} / \mathrm{ml}$ of IS solution). After being vortexed for $1 \mathrm{~min}$, the mixture was centrifuged at $14500 \times \mathrm{g}$ for $15 \mathrm{mins}$ at room temperature. Then, $100 \mu \mathrm{L}$ of the supernatant solution was transferred to a $1.5 \mathrm{~mL}$ Eppendorf tube prior to adding $200 \mu \mathrm{L}$ mobile phase (A : B, $70: 30, \mathrm{~V} / \mathrm{V})$. The sample was centrifuged at $14500 \mathrm{rpm}$ for another $15 \mathrm{mins}$ after being vortexed for $1 \mathrm{~min}$ at room temperature. Then, $5 \mu \mathrm{L}$ of the supernatant solution was directly injected into the HPLC-MS/MS system for analysis.

2.7. Human Sample. This research was approved by and performed at Changzheng Hospital (Shanghai, China) from March 2019 to May 2019. Blood samples were collected in EDTA-3K tubes from NSCLC patients after treatment with TKIs, and a total of 25 patients with NSCLC were enrolled in this study. And $3 \mathrm{~mL}$ venous blood samples were collected after a food fasting overnight, gently mixed after the TKI in NSCLC patients had reached steady concentration (two weeks after the first dose), and then centrifuged at $3000 \times \mathrm{g}$ for 10 minutes. The plasma was harvested and measured by the above method.

2.8. Method Validation. According to the Chinese Pharmacopoeia (version 2010) and FDA guidelines [16], method validation includes specificity, lower limit of quantitation (LLOQ), linearity, inter- and intraprecision and accuracy, carryover, extraction recovery, matrix effect, and stability.

\section{Results and Discussion}

3.1. Chromatography Condition Optimization. In this experiment, some universal columns containing Agilent Zorbax SB-C18 $(2.1 \mathrm{~mm} \times 100 \mathrm{~mm}, 3.5 \mu \mathrm{m})$, Waters XSELECT $^{\mathrm{TM}}$ HSS PFP $(2.1 \mathrm{~mm} \times 100 \mathrm{~mm}, 3.5 \mu \mathrm{m})$, and XBridge $^{\mathrm{TM}}$ BEH C18 $(2.1 \mathrm{~mm} \times 50 \mathrm{~mm}, 2.5 \mu \mathrm{m})$ were tested for their retention and separation ability. By comparing their chromatographic features (such as resolution, retention time, response value, and peak shape), the results showed a better peak shape and response on the Agilent Zorbax SBC18 $(2.1 \mathrm{~mm} \times 100 \mathrm{~mm}, 3.5 \mu \mathrm{m})$ column and achieved complete separation of analytes in a short time from endogenous interferents. Therefore, the Agilent Zorbax SBC18 $(2.1 \mathrm{~mm} \times 100 \mathrm{~mm}, 3.5 \mu \mathrm{m})$ column was selected for the development of the method.
Different mobile phase additives (such as formic acid and ammonium acetate) were added to improve the peak shape, response, and retention time. When ammonium acetate was added to the mobile phase, the responses of all analytes were low, and the peak shape was poor. And then, retention time and separation effect were gained after testing with different ratios of formic acid (FA) $(0.05 \%, 0.1 \%$, and $0.2 \% \mathrm{FA}$ ) in the mobile phase. It was found that when $0.1 \%$ FA was added to the water phase, the response of analytes could be significantly increased.

3.2. Sample Pretreatment Optimization. Protein precipitation, solid-phase extraction (SPE), and liquid-liquid extraction are the main methods used in laboratory sample pretreatment. The protein precipitation method is a simple, economical, and time-saving method, while the cost of liquid-liquid extraction and solid-phase extraction is relatively higher. Therefore, the protein precipitation method is first tested. Acetonitrile and methanol were used as precipitating agents to remove protein from plasma. By comparing the extraction recovery and matrix effect of acetonitrile and methanol in different proportions, it was found that the ratio of acetonitrile to the sample is $2: 1$, and the extraction recovery is higher. In order to further purify the sample and reduce impurities in the sample, the supernatant after centrifugation was further treated. An equal volume of aqueous phase, organic phase, and their mixtures in different ratios was added to the supernatant. Results showed that the ratio of the mixed mobile phase is $7: 3(\mathrm{~V}$ : $\mathrm{V})$, and the extraction recovery and peak shape are relatively ideal. In the end, comparing the extraction recovery and matrix effect of the supernatant and mixed mobile phase in different proportions, results found that the ratio of the mixed mobile phase to the supernatant is $2: 1(\mathrm{~V}: \mathrm{V})$, and the extraction recovery is higher. The pretreatment method is simple and economical, which can basically meet the requirements of this experiment and has good practicability.

\subsection{Method Validation}

3.3.1. Specificity. Comparisons of specificity from blank, IS, and six analytes spiked and clinical real samples (Figure 2) indicated no significant interferences at the same retentiontimes of the analytes and IS. The retention time of six TKIs and IS is as follows: IS: $5.2 \mathrm{~min}$, iconitib: $2.7 \mathrm{~min}$, erlotinib: $4.2 \mathrm{~min}$, gefitinib: $1.3 \mathrm{~min}$, crizotinib: $1.3 \mathrm{~min}$, afatinib: $1.3 \mathrm{~min}$, and osimertinib: $1.9 \mathrm{~min}$. 


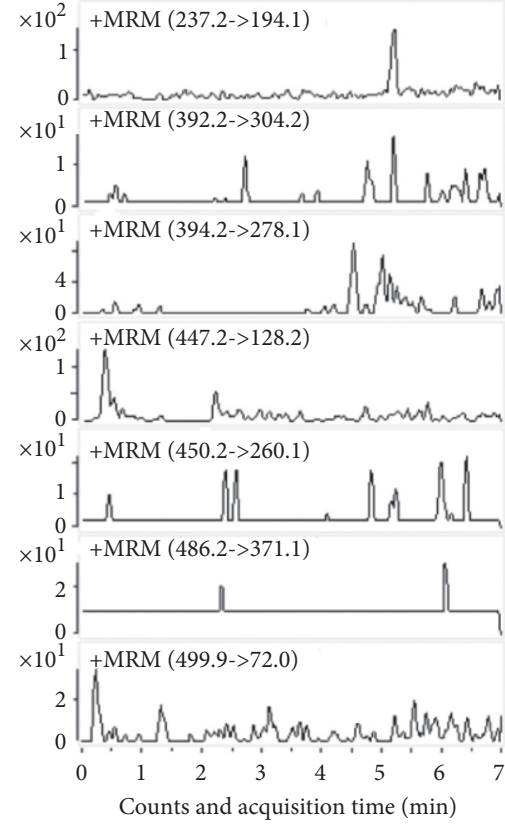

(a)

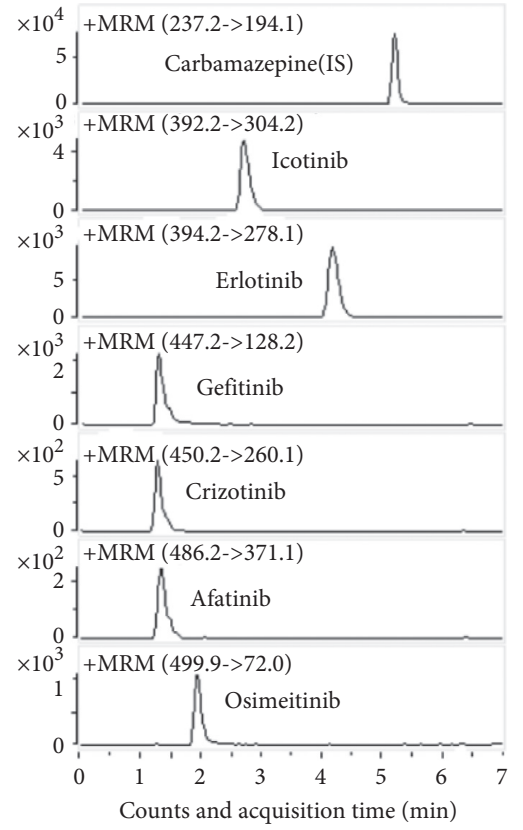

(b)

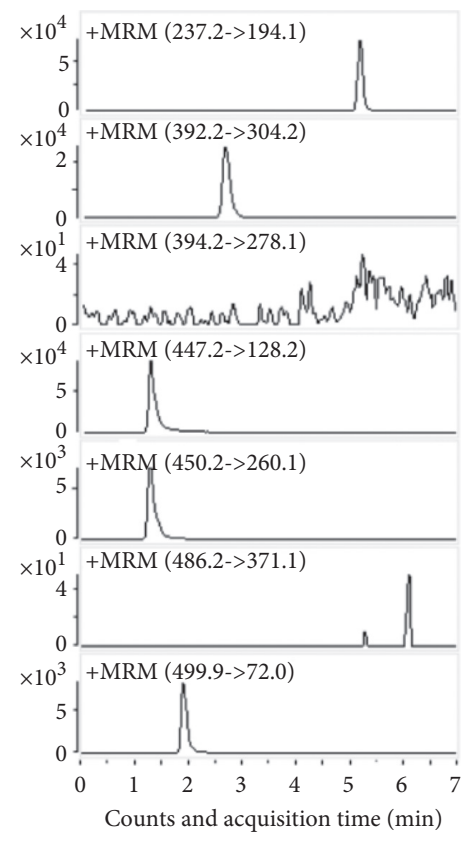

(c)

FIGURE 2: Representative MRM chromatograms of six TKIs: (a) blank sample; (b) blank sample spiked with the LLOQ concentration of six TKIs and IS; (c) real sample concentration (icotinib: $504 \mathrm{ng} / \mathrm{mL}$, erlotinib: ND, gefitinib: $623 \mathrm{ng} / \mathrm{mL}$, crizotinib: $327 \mathrm{ng} / \mathrm{mL}$, afatinib: ND, osimertinib: $250 \mathrm{ng} / \mathrm{mL}$, and IS: $100 \mathrm{ng} / \mathrm{mL})$. ND = not detected.

TABLE 2: Linearity regression parameters of six analytes.

\begin{tabular}{lcccc}
\hline Analytes & Regression type & Linear range & Weighing factor & Regression equations \\
\hline Gefitinib & Linearity & $10.0-1000.0$ & $1 / \chi^{2}$ & $y=0.0025 * x-0.0029$ \\
Icotinib & Linearity & $50.0-4000.0$ & $1 / \chi^{2}$ & $y=0.0011 * x-0.0129$ \\
Erlotinib & Linearity & $50.0-4000.0$ & $1 / \chi^{2}$ & $y=0.0024 * x-0.0275$ \\
Crizotinib & Linearity & $10.0-1000.0$ & $1 / \chi^{2}$ & $y=0.0193 * x-0.4494$ \\
Osimertinib & Linearity & $10.0-1000.0$ & $1 / \chi^{2}$ & $y=0.0016 * x-0.0023$ \\
Afatinib & Linearity & $5.0-400.0$ & $1 / \chi^{2}$ & 0.9940 \\
\hline
\end{tabular}

3.3.2. Linearity. Through the construction of calibration curves, the linear correlation coefficients $(R)$ of all analytes were greater than 0.990 under the weighing coefficient of $1 /$ $\chi^{2}$. The linear correlation coefficients $(R)$ were more than 0.990 for all analytes. Results of regression equations for the calibration curves are presented in Table 2. The LLOQ was $10 \mathrm{ng} / \mathrm{mL}$ in the human plasma matrix of gefitinib, crizotinib, and osimertinib, $50 \mathrm{ng} / \mathrm{mL}$ in iconitib and erlotinib, and $5 \mathrm{ng} / \mathrm{mL}$ in afatinib, which were also in accordance with the accuracy within $\pm 20 \%$ and precision less than $20 \%$. The LLOQ and accuracy assessment results are summarized in Table 3.

3.3.3. Matrix Effect and Extraction Recovery. The matrix effect and extraction recovery of samples (low, middle, and high) were investigated. The results showed that the matrix effect of the analytes was between $96.83 \%$ and $114.09 \%$, and the recovery was between $76.66 \%$ and $97.18 \%$. The RSD (\%) of the matrix effect and extraction recovery factors was less than $15 \%$. The results are shown in Table 4 .
3.3.4. Precision and Accuracy. Three levels of QC samples (low, middle, and high) were chosen to analyze the interand intra-accuracy and precision. The results of accuracy with inter- and intra-accuracy were $85.36 \%$ to $111.38 \%$ and $85.24 \%$ to $113.04 \%$, and the inter- and intraprecision were $1.02 \%$ to $5.41 \%$ and $0.33 \%$ to $4.97 \%$. Table 5 summarizes the inter- and intraday accuracy and precision for the analytes.

3.3.5. Stability. Short-term stability $\left(25^{\circ} \mathrm{C}\right.$ in room temperature for $12 \mathrm{~h}$ and in the autosampler for $24 \mathrm{~h}$ ), long-term stability $\left(1\right.$ month at $\left.-80^{\circ} \mathrm{C}\right)$, and three freeze-thaw cycles' stability were determined for all analytes in triplicate at each of the low, middle, and high concentrations. Results of stability are shown in Table 6.

3.4. Application of Clinical Samples Treated by TKIs. To test the applicability of this method, 25 plasma samples were collected from 25 NSCLC patients who were treated with one of six drugs. Gefitinib (250 mg qd), erlotinib (150 mg 
TABle 3: Precision and accuracy of the LLOQ of six analytes' calibration curves.

\begin{tabular}{lcccc}
\hline Analytes & Nominal concentration $(\mathrm{ng} / \mathrm{mL})$ & Measured concentration $(\mathrm{ng} / \mathrm{ml}) \pm$ SD & Precision RSD (\%) & Accuracy mean (\%) \\
\hline Gefitinib & 10 & $8.82 \pm 0.29$ & 6.31 & 96.22 \\
Icotinib & 50 & $50.22 \pm 0.91$ & 1.81 & 1.87 \\
Erlotinib & 50 & $49.01 \pm 0.92$ & 7.27 & 98.44 \\
Crizotinib & 10 & $10.07 \pm 0.36$ & 4.31 & 101.39 \\
Osimertinib & 10 & $9.75 \pm 0.21$ & 5.76 & 95.15 \\
Afatinib & 5 & $5.30 \pm 0.31$ & & 105.98 \\
\hline
\end{tabular}

TABle 4: Extraction recovery and matrix effect of six analytes (\%) $(n=3)$.

\begin{tabular}{|c|c|c|c|c|c|}
\hline \multirow{2}{*}{ Analytes } & \multirow{2}{*}{ Nominal concentration $(\mathrm{ng} / \mathrm{mL})$} & \multicolumn{2}{|c|}{ Extraction recovery } & \multicolumn{2}{|c|}{ Matrix effect } \\
\hline & & Mean $(\%) \pm S D$ & RSD (\%) & Mean $(\%) \pm S D$ & RSD (\%) \\
\hline \multirow{3}{*}{ Gefitinib } & 20 & $91.18 \pm 3.49$ & \multirow{3}{*}{3.08} & $114.09 \pm 7.4$ & \multirow{3}{*}{2.56} \\
\hline & 100 & $80.75 \pm 3.34$ & & $113.83 \pm 8.88$ & \\
\hline & 500 & $97.18 \pm 1.13$ & & $108.98 \pm 11.45$ & \\
\hline \multirow{3}{*}{ Icotinib } & 100 & $81.41 \pm 1.33$ & \multirow{3}{*}{3.11} & $107.58 \pm 4.28$ & \multirow{3}{*}{3.49} \\
\hline & 500 & $93.89 \pm 3.91$ & & $100.91 \pm 2.91$ & \\
\hline & 2000 & $96.02 \pm 1.62$ & & $101.85 \pm 5.85$ & \\
\hline \multirow{3}{*}{ Erlotinib } & 100 & $85.24 \pm 3.33$ & \multirow{3}{*}{3.00} & $108.67 \pm 8.22$ & \multirow{3}{*}{5.49} \\
\hline & 500 & $90.10 \pm 1.8$ & & $101.26 \pm 2.47$ & \\
\hline & 2000 & $85.85 \pm 1.99$ & & $97.63 \pm 5.90$ & \\
\hline \multirow{3}{*}{ Crizotinib } & 20 & $76.66 \pm 6.33$ & \multirow{3}{*}{2.71} & $109.91 \pm 12.21$ & \multirow{3}{*}{5.31} \\
\hline & 100 & $77.75 \pm 5.15$ & & $100.98 \pm 7.88$ & \\
\hline & 500 & $82.10 \pm 3.87$ & & $96.83 \pm 11.76$ & \\
\hline \multirow{3}{*}{ Osimertinib } & 20 & $82.25 \pm 7.63$ & \multirow{3}{*}{2.92} & $100.58 \pm 4.94$ & \multirow{3}{*}{2.97} \\
\hline & 100 & $90.53 \pm 2.48$ & & $102.72 \pm 5.27$ & \\
\hline & 500 & $81.76 \pm 4.03$ & & $96.85 \pm 10.60$ & \\
\hline \multirow{3}{*}{ Afatinib } & 10 & $85.20 \pm 1.38$ & \multirow{3}{*}{3.12} & $102.56 \pm 17.39$ & \multirow{3}{*}{2.91} \\
\hline & 50 & $90.55 \pm 8.06$ & & $108.45 \pm 15.34$ & \\
\hline & 200 & $95.69 \pm 8.61$ & & $107.08 \pm 9.03$ & \\
\hline
\end{tabular}

RSD was calculated using the IS-normalized matrix and recovery factors.

TABLE 5: Inter- and intra-accuracy and precision of six analytes $(n=5)$.

\begin{tabular}{|c|c|c|c|c|c|c|c|}
\hline \multirow[b]{2}{*}{ Analytes } & \multirow[b]{2}{*}{$\begin{array}{l}\text { Nominal } \\
\text { concentration }(\mathrm{ng} / \mathrm{ml})\end{array}$} & \multicolumn{3}{|c|}{ Interday } & \multicolumn{3}{|c|}{ Intraday } \\
\hline & & $\begin{array}{l}\text { Measured concentration } \\
(\text { mean } \pm S D)\end{array}$ & $\begin{array}{l}\text { Accuracy } \\
(\mathrm{RE} \%)\end{array}$ & $\begin{array}{l}\text { Precision } \\
(\text { RSD \%) }\end{array}$ & $\begin{array}{l}\text { Measured concentration } \\
(\text { mean } \pm S D)\end{array}$ & $\begin{array}{l}\text { Accuracy } \\
(\mathrm{RE} \%)\end{array}$ & $\begin{array}{l}\text { Precision } \\
(\mathrm{RSD} \%)\end{array}$ \\
\hline \multirow{3}{*}{ Gefitinib } & 20 & $17.70 \pm 0.66$ & -11.5 & 3.75 & $17.32 \pm 0.26$ & -13.4 & 1.51 \\
\hline & 100 & $87.81 \pm 1.81$ & -12.19 & 2.06 & $87.77 \pm 2.22$ & -12.23 & 2.53 \\
\hline & 500 & $552.89 \pm 15.82$ & 10.59 & 2.86 & $565.21 \pm 9.26$ & 13.04 & 1.63 \\
\hline \multirow{3}{*}{ Icotinib } & 100 & $91.09 \pm 0.93$ & -8.91 & 1.02 & $91.56 \pm 0.49$ & -8.44 & 0.53 \\
\hline & 500 & $439.77 \pm 12.18$ & -12.05 & 2.77 & $426.70 \pm 1.82$ & -14.66 & 0.42 \\
\hline & 2000 & $1919.82 \pm 39.30$ & -0.40 & 2.04 & $1966.42 \pm 27.49$ & -1.68 & 1.39 \\
\hline \multirow{3}{*}{ Erlotinib } & 100 & $87.59 \pm 1.14$ & -12.41 & 1.31 & $88.94 \pm 0.82$ & -11.06 & 0.93 \\
\hline & 500 & $441.47 \pm 6.58$ & -11.71 & 1.50 & $450.70 \pm 1.51$ & -9.86 & 0.33 \\
\hline & 2000 & $1887.25 \pm 94.36$ & -5.64 & 2.19 & $1933.51 \pm 37.33$ & -3.25 & 1.93 \\
\hline \multirow{3}{*}{ Crizotinib } & 20 & $18.17 \pm 0.62$ & -9.15 & 3.42 & $18.55 \pm 0.92$ & -7.25 & 4.97 \\
\hline & 100 & $91.67 \pm 3.11$ & -8.33 & 3.39 & $88.67 \pm 1.71$ & -11.33 & 1.92 \\
\hline & 500 & $538.54 \pm 12.68$ & 7.71 & 2.35 & $538.57 \pm 4.57$ & 7.71 & 0.85 \\
\hline \multirow{3}{*}{ Osimertinib } & 20 & $18.72 \pm 0.92$ & -6.40 & 4.92 & $18.52 \pm 0.46$ & -7.40 & 2.53 \\
\hline & 100 & $85.36 \pm 2.96$ & -14.64 & 3.47 & $85.24 \pm 4.95$ & -14.76 & 5.81 \\
\hline & 500 & $556.91 \pm 11.80$ & 11.38 & 2.12 & $561.76 \pm 15.24$ & 12.35 & 2.71 \\
\hline \multirow{3}{*}{ Afatinib } & 10 & $9.92 \pm 0.53$ & -0.80 & 5.41 & $9.76 \pm 0.19$ & -2.40 & 1.94 \\
\hline & 50 & $53.78 \pm 2.28$ & 7.56 & 4.24 & $51.54 \pm 1.74$ & 3.08 & 3.37 \\
\hline & 200 & $217.83 \pm 4.73$ & 8.92 & 2.17 & $220.65 \pm 4.71$ & 10.33 & 2.13 \\
\hline
\end{tabular}




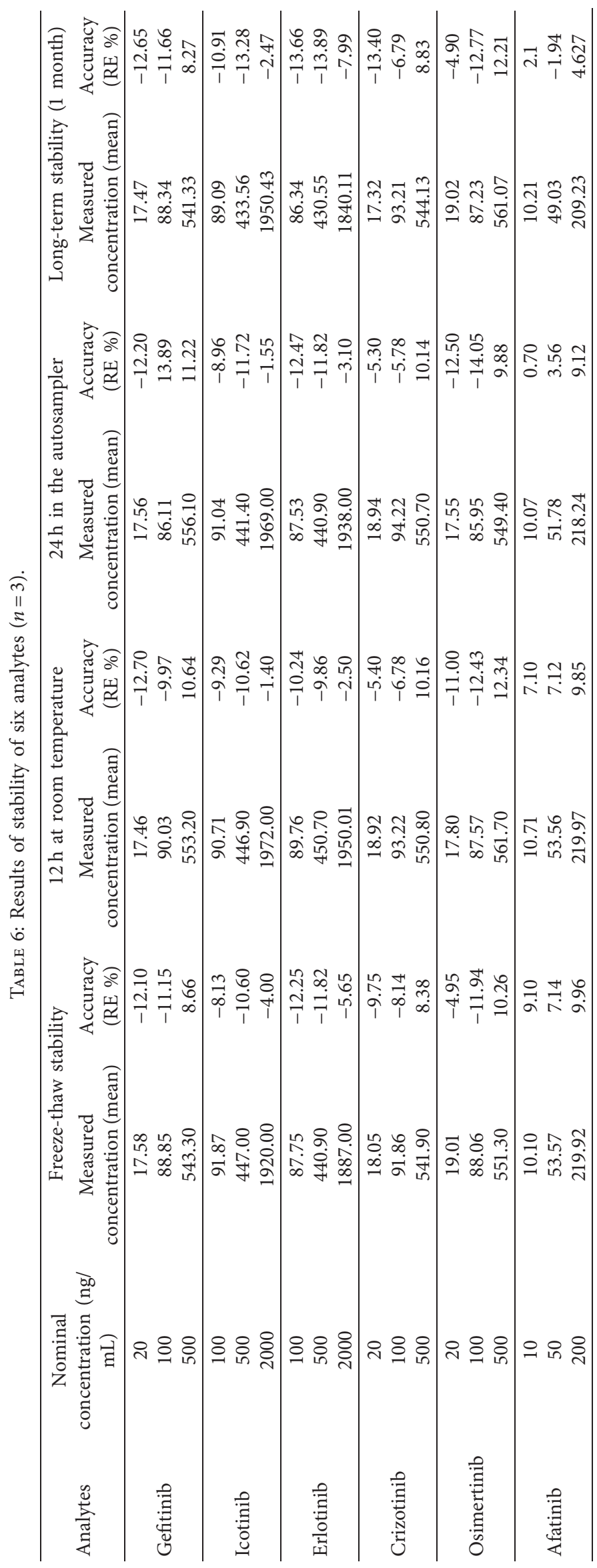




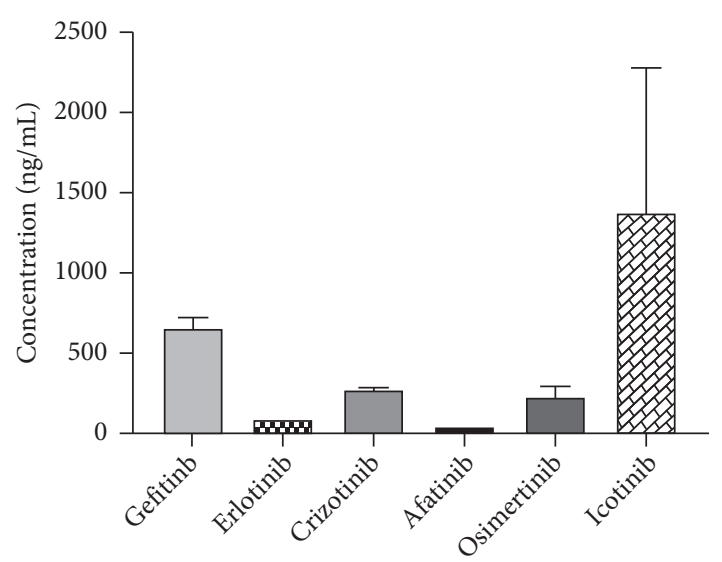

FIGURE 3: Drug concentration distribution of NSCLC patients with 25 cases.

qd), icotinib (125 mg tid), afatinib (40 mg qd), osimertinib (80 $\mathrm{mg} \mathrm{qd}$ ), and crizotinib (250 $\mathrm{mg}$ bid) at standard doses at Changzheng Hospital were prescribed according to clinical diagnosis. Nine (erlotinib), seven (osimertinib), three (gefitinib), three (crizotinib), two (afatinib), and one (erlotinib) plasma samples were collected from patients, and the time points were designed in 7-14 days after administration (trough concentration). The TKIs were quantitatively measured in the patients' plasma. Results of the drug concentration distribution of TKIs in NSCLC patients are shown in Figure 3.

The drug exposure in vivo has a close relation with the treatment efficacy and/or adverse reaction, and it was still the key point for the clinical optimization of drug dose [17-21]. There were differences in drug absorption, distribution, metabolism, and excretion between patients and within patients, and several studies in recent years were also reported [22-24]. This makes monitoring the concentration of TKI drugs' exposure in vivo particularly significant, and the method is an appropriate effective detection technology to increase the treatment efficacy and/or adverse reaction in the process of individualized dosing administration. Due to the limitation of objective conditions, the number of enrolled patients was small, due to which the relationship between the exposure and treatment efficacy could not be determined.

\section{Conclusion}

A simple, rapid, and sensitive method for the simultaneous determination of TKIs (including gefitinib, erlotinib, crizotinib, afatinib, osimertinib, and icotinib) in human plasma from NSCLC patients by the HPLC-MS/MS method was developed and validated. The analytical time was $7 \mathrm{~min}$ for six analytes after optimizing detection conditions, and the sample pretreatment method was simple, rapid, and economical. This method was suitable for clinical therapeutic drug monitoring to obtain a better treatment outcome.

\section{Data Availability}

All data are included within the article and the supplementary materials.

\section{Conflicts of Interest}

The authors declare that they have no conflicts of interest.

\section{Authors' Contributions}

Yanping Liu and Hua Liu contributed equally to this work.

\section{Acknowledgments}

This work was supported by the National Key R\&D Program of China (no. 2018YFC1707304).

\section{Supplementary Materials}

To test the applicability of this method, 25 plasma samples were collected from 25 NSCLC patients who were treated with one of the six TKIs. Icotinib $(n=9)$, osimertinib $(n=7)$, gefitinib $(n=3)$, crizotinib $(n=3)$, afatinib $(n=2)$, and erlotinib $(n=1)$ were collected from clinical therapeutics patients. The data results of drug valley concentration values in human plasma of six TKIs are shown as supplementary materials. Table 1: plasma concentration of TKIs was determined by oral administration. (Supplementary Materials)

\section{References}

[1] F. Bray, J. Ferlay, I. Soerjomataram, R. L. Siegel, L. A. Torre, and A. Jemal, "Global cancer statistics 2018: GLOBOCAN estimates of incidence and mortality worldwide for 36 cancers in 185 countries," CA: A Cancer Journal for Clinicians, vol. 68, no. 6, pp. 394-424, 2018.

[2] H. Wakelee, K. Kelly, and M. J. Edelman, "50 years of progress in the systemic therapy of non-small cell lung cancer," American Society of Clinical Oncology Educational Book, vol. 34, pp. 177-189, 2014.

[3] A. Muth, N. S. GKulkarni, V. Parvathaneni et al., "Tyrosine kinase inhibitor conjugated quantum dots for non-small cell lung cancer (NSCLC) treatment," European Journal of Pharmaceutical Sciences, vol. 133, pp. 145-159, 2019.

[4] J. A. Barta, C. A. Powell, and J. P. Wisnivesky, "Global epidemiology of lung cancer," Annals of Global Health, vol. 85, no. 1, p. 8, 2019.

[5] N. Girard, "Optimizing outcomes in EGFR mutation-positive NSCLC: which tyrosine kinase inhibitor and when?” Future Oncology, vol. 14, no. 11, pp. 1117-1132, 2018.

[6] A. T. Shaw, D.-W. Kim, K. Nakagawa et al., "Crizotinib versus chemotherapy in advanced ALK-positive lung cancer," New England Journal of Medicine, vol. 368, no. 25, pp. 2385-2394, 2013.

[7] H. Yu, N. Steeghs, C. M. Nijenhuis, J. H. M. Schellens, J. H. Beijnen, and A. D. R. Huitema, "Practical guidelines for therapeutic drug monitoring of anticancer tyrosine kinase inhibitors: focus on the pharmacokinetic targets," Clinical Pharmacokinetics, vol. 53, no. 4, pp. 305-325, 2014.

[8] H. Hayashi, Y. Kita, H. Iihara et al., "Simultaneous and rapid determination of gefitinib, erlotinib and afatinib plasma levels using liquid chromatography/tandem mass spectrometry in patients with non-small-cell lung cancer," Biomedical Chromatography, vol. 30, no. 7, pp. 1150-1154, 2016.

[9] C. J. Lucas and J. H. Martin, "Pharmacokinetic-guided dosing of new oral cancer agents," The Journal of Clinical Pharmacology, vol. 57, no. Suppl 10, pp. S78-S98, 2017. 
[10] S. Wind, D. Schnell, T. Ebner, M. Freiwald, and P. Stopfer, "Clinical pharmacokinetics and pharmacodynamics of afatinib," Clinical Pharmacokinetics, vol. 56, no. 3, pp. 235-250, 2017.

[11] I. Solassol, F. Pinguet, and X. Quantin, "FDA-and EMAapproved tyrosine kinase inhibitors in advanced EGFR-mutated non-small cell lung cancer: safety, tolerability, plasma concentration monitoring, and management," Biomolecules, vol. 9, no. 11, p. 668, 2019.

[12] H. H. Huynh, C. Pressiat, H. Sauvageon et al., "Development and validation of a simultaneous quantification method of 14 tyrosine kinase inhibitors in human plasma using LC-MS/ MS,” Therapeutic Drug Monitoring, vol. 39, no. 1, pp. 43-54, 2017.

[13] R. Reis, L. Labat, M. Allard et al., "Liquid chromatographytandem mass spectrometric assay for therapeutic drug monitoring of the EGFR inhibitors afatinib, erlotinib and osimertinib, the ALK inhibitor crizotinib and the VEGFR inhibitor nintedanib in human plasma from non-small cell lung cancer patients," Journal of Pharmaceutical and Biomedical Analysis, vol. 158, pp. 174-183, 2018.

[14] C. Merienne, M. Rousset, D. Ducint et al., "High throughput routine determination of 17 tyrosine kinase inhibitors by LCMS/MS," Journal of Pharmaceutical and Biomedical Analysis, vol. 150, pp. 112-120, 2018.

[15] Y. He, L. Zhou, S. Gao et al., "Development and validation of a sensitive LC-MS/MS method for simultaneous determination of eight tyrosine kinase inhibitors and its application in mice pharmacokinetic studies," Journal of Pharmaceutical and Biomedical Analysis, vol. 148, pp. 65-72, 2018.

[16] Z. Wang, Y. Yang, F. Zhang et al., "A direct, sensitive and efficient method for determination of alpha-fluoro-beta-alanine in urine: evaluating the influence of magnesium isoglycyrrhizinate on excretion in rat model," Journal of Chromatography B, vol. 1102-1103, pp. 17-22, 2018.

[17] T. Kawata, M. Higashimori, Y. Itoh et al., "Gefitinib exposure and occurrence of interstitial lung disease in Japanese patients with non-small-cell lung cancer," Cancer Chemotherapy and Pharmacology, vol. 83, no. 5, pp. 849-858, 2019.

[18] N. Ueno, S. Banno, Y. Endo et al., "Treatment status and safety of crizotinib in 2028 Japanese patients with ALK-positive NSCLC in clinical settings," Japanese Journal of Clinical Oncology, vol. 49, no. 7, pp. 676-686, 2019.

[19] K. Nakao, S. Kobuchi, S. Marutani et al., "Population pharmacokinetics of afatinib and exposure-safety relationships in Japanese patients with EGFR mutation-positive non-small cell lung cancer," Scientific Reports, vol. 9, no. 1, p. 18202, 2019.

[20] A. Almomen, H. M. Maher, N. Z. Alzoman, S. M. Shehata, and A. Alsubaie, "Flavoured water consumption alters pharmacokinetic parameters and increases exposure of erlotinib and gefitinib in a preclinical study using wistar rats," PeerJ, vol. 8, p. e9881, 2020.

[21] S. L. Groenland, D. R. Geel, J. M. Janssen et al., "Exposureresponse analyses of anaplastic lymphoma kinase inhibitors crizotinib and alectinib in non-small cell lung cancer patients," Clinical Pharmacology \& Therapeutics, vol. 109, no. 2, pp. 394-402, 2020.

[22] W. Zhang, Y. Zhang, Q. Zhao et al., "Long-term safety of icotinib in patients with non-small cell lung cancer: a retrospective, real-world study," Journal of Thoracic Disease, vol. 12, no. 3, pp. 639-650, 2020.

[23] H. Zhang, Q. Li, X. Zhu et al., "Association of variability and pharmacogenomics with bioequivalence of gefitinib in healthy male subjects," Frontiers in Pharmacology, vol. 9, p. 849, 2018.
[24] S. Sakamoto, K. Sato, Y. Takita et al., "ABCG2 C421A polymorphisms affect exposure of the epidermal growth factor receptor inhibitor gefitinib," Investigational New Drugs, vol. 38, no. 6, pp. 1687-1695, 2020. 